\title{
Discrete maximum principle and a Delaunay-type mesh condition for linear finite element approximations of two-dimensional anisotropic diffusion problems
}

\author{
Weizhang Huang *
}

\begin{abstract}
The finite element solution of two-dimensional anisotropic diffusion problems is considered. A Delaunay-type mesh condition is developed for linear finite element approximations to satisfy a discrete maximum principle. The condition is shown to be weaker than the existing anisotropic non-obtuse angle condition. It reduces to the well known Delaunay condition for the special case with the identity diffusion matrix. Numerical results are presented to verify the theoretical findings.
\end{abstract}

AMS 2010 Mathematics Subject Classification. 65N30, 65N50

Key words. anisotropic diffusion, discrete maximum principle, finite element, mesh generation, Delaunay triangulation, Delaunay condition

\section{Introduction}

We are concerned with the linear finite element (FEM) solution of the two-dimensional anisotropic diffusion equation

$$
-\nabla \cdot(\mathbb{D} \nabla u)=f, \quad \text { in } \quad \Omega
$$

subject to the Dirichlet boundary condition

$$
u=g, \quad \text { on } \quad \partial \Omega
$$

where $\Omega \in \mathbb{R}^{2}$ is a connected polygonal domain, $f$ and $g$ are given functions, and $\mathbb{D}=\mathbb{D}(x, y)$ is the diffusion matrix assumed to be symmetric and strictly positive definite on $\Omega$. This boundary value problem (BVP) is a model of anisotropic diffusion problems arising in various fields such as plasma physics [15, 16, 17, 34, 36, 38], petroleum reservoir simulation [1, 2, 10, 13, 32, and image processing [6, 7, 21, 33, 35, 43. A distinct feature of the BVP is that its solution satisfies the maximum principle and is monotone when $f(x, y) \leq 0$ for all $(x, y) \in \Omega$. A challenge in the numerical solution of the BVP is to design a scheme so that the resulting numerical approximations satisfy a discrete maximum principle (DMP).

* Department of Mathematics, the University of Kansas, Lawrence, KS 66045, U.S.A. (huang@math.ku.edu). The work was supported in part by the National Science Foundation (USA) under Grant DMS-0712935. 
Development of DMP satisfaction schemes for solving diffusion problems has attracted considerable interest in the past; e.g., see [4, 5, 8, 9, 18, 19, 20, 23, 27, 39, 40, 41, 42, 44, for isotropic diffusion problems where $\mathbb{D}=a(x, y) I$ with $a(x, y)$ being a scaler function and [10, 11, 15, 16, 17, 22, 24, 25, 26, 28, 29, 30, 31, 32, 36] for anisotropic diffusion problems where $\mathbb{D}(x, y)$ can be heterogeneous and anisotropic. For example, Ciarlet and Raviart 9] (also see Brandts et al. [4]) show that the linear finite element method for an isotropic diffusion problem satisfies DMP when the mesh is simplicial and satisfies the non-obtuse angle condition requiring the dihedral angles of mesh elements to be non-obtuse. In two dimensions and for the special case $\mathbb{D}=I$, the condition can be replaced by the Delaunay condition, a weaker condition that only requires the sum of any pair of angles opposite a common edge to be less than or equal to $\pi$ [27, 41]. Moreover, Xu and Zikatanov [44] show that the non-obtuse angle condition at edges where the diffusion coefficient is discontinuous and the Delaunay condition at other places guarantee DMP satisfaction. Recently, $\mathrm{Li}$ and Huang [28] generalize the non-obtuse angle condition to anisotropic diffusion problems and obtain the so-called anisotropic non-obtuse angle condition requiring the dihedral angles of mesh elements to be non-obtuse when measured in a metric depending on $\mathbb{D}$.

The objective of this paper is to extend the Delaunay condition to anisotropic diffusion problems. A Delaunay-type mesh condition is developed for the DMP satisfaction of linear finite element approximations for those problems. It is shown that the new condition reduces to the Delaunay condition for the special case $\mathbb{D}=I$ and is weaker than the anisotropic non-obtuse angle condition developed in [28]. We attain the new condition by investigating the stiffness matrix as a whole. This is different from [28] where only local stiffness matrices on individual elements are considered. The main theoretical result is given in Theorem 4.1 .

This paper is organized as follows. The linear finite element formulation for BVP (1) and (2) is given in Section 2. Section 3 is devoted to the description and geometric interpretation of the anisotropic non-obtuse angle condition. The Delaunay-type mesh condition is developed in Section 4, followed by Section 5 with numerical results verifying the theoretical findings. Finally, Section 6 contains conclusions and comments.

\section{Linear finite element formulation for the model problem}

Consider the linear finite element solution of BVP (1) and (2). Assume that a family of triangular meshes $\left\{\mathcal{T}_{h}\right\}$ is given for $\Omega$. Let

$$
U_{g}=\left\{v \in H^{1}(\Omega)|v|_{\partial \Omega}=g\right\} .
$$

Denote by $U_{g^{h}}^{h} \subset U_{g}$ the linear finite element space associated with mesh $\mathcal{T}_{h}$, where $g^{h}$ is a linear approximation to $g$ on the boundary. A linear finite element solution $\tilde{u}^{h} \in U_{g^{h}}^{h}$ to BVP (1) and (2) is defined by

$$
\sum_{K \in \mathcal{T}_{h}} \int_{K}\left(\nabla v^{h}\right)^{T} \mathbb{D} \nabla \tilde{u}^{h} d x d y=\sum_{K \in \mathcal{T}_{h}} \int_{K} f v^{h} d x d y, \quad \forall v^{h} \in U_{0}^{h}
$$

where $U_{0}^{h}=U_{g^{h}}^{h}$ with $g^{h}=0$. Generally speaking, the integrals in 3 cannot be carried out analytically and numerical quadrature is often necessary. We assume that a quadrature rule has 
been chosen on the reference element $\hat{K}$,

$$
\int_{\hat{K}} v(\xi, \eta) d \xi d \eta \approx|\hat{K}| \sum_{k=1}^{m} \hat{w}_{k} v\left(\hat{b}_{k}\right), \quad \sum_{k=1}^{m} \hat{w}_{k}=1,
$$

where $\hat{w}_{k}$ 's are the weights and $\hat{b}_{k}$ 's are the quadrature nodes. Many quadrature rules can be used for this purpose; e.g., see [12]. An example is $\hat{w}_{k}=\frac{1}{3}(k=1,2,3)$ and the barycentric coordinates of the nodes $\left(\frac{1}{6}, \frac{1}{6}, \frac{2}{3}\right),\left(\frac{1}{6}, \frac{2}{3}, \frac{1}{6}\right)$, and $\left(\frac{2}{3}, \frac{1}{6}, \frac{1}{6}\right)$.

Let $F_{K}$ be the affine mapping from $\hat{K}$ to $K$ such that $K=F_{K}(\hat{K})$, and denote $b_{k}^{K}=F_{K}\left(\hat{b}_{k}\right)$, $k=1, \cdots, m$. Upon applying (4) to the integrals in (3) and changing variables, the finite element approximation problem becomes seeking $u^{h} \in U_{g^{h}}^{h}$ such that

$$
\left.\sum_{K \in \mathcal{T}_{h}}|K| \sum_{k=1}^{m} \hat{w}_{k}\left(\left.\nabla v^{h}\right|_{K}\right)^{T} \mathbb{D}\left(b_{k}^{K}\right) \nabla u^{h}\right|_{K}=\sum_{K \in \mathcal{T}_{h}}|K| \sum_{k=1}^{m} \hat{w}_{k} f\left(b_{k}^{K}\right) v^{h}\left(b_{k}^{K}\right), \quad \forall v^{h} \in U_{0}^{h}
$$

where $\left.\nabla v^{h}\right|_{K}$ and $\left.\nabla u^{h}\right|_{K}$ denote the restriction of $\nabla v^{h}$ and $\nabla u^{h}$ on $K$, respectively. We have used the fact that $\left.\nabla v^{h}\right|_{K}$ and $\left.\nabla u^{h}\right|_{K}$ are constant in deriving (5). Let

$$
\mathbb{D}_{K}=\sum_{k=1}^{m} \hat{w}_{k} \mathbb{D}\left(b_{k}^{K}\right)
$$

Obviously, $\mathbb{D}_{K}$ is an average of $\mathbb{D}$ on $K$. Eq. (5) can be written into

$$
\left.\sum_{K \in \mathcal{T}_{h}}|K|\left(\left.\nabla v^{h}\right|_{K}\right)^{T} \mathbb{D}_{K} \nabla u^{h}\right|_{K}=\sum_{K \in \mathcal{T}_{h}}|K| \sum_{k=1}^{m} \hat{w}_{k} f\left(b_{k}^{K}\right) v^{h}\left(b_{k}^{K}\right), \quad \forall v^{h} \in U_{0}^{h} .
$$

We now express (7) in a matrix form. Denote the numbers of the elements, vertices, and interior vertices of mesh $\mathcal{T}_{h}$ by $N, N_{v}$, and $N_{v i}$, respectively. Assume that the vertices are ordered in such a way that the first $N_{v i}$ vertices are the interior vertices. Then $U_{0}^{h}$ and $u^{h}$ can be expressed as

$$
\begin{gathered}
U_{0}^{h}=\operatorname{span}\left\{\phi_{1}, \cdots, \phi_{N_{v i}}\right\}, \\
u^{h}=\sum_{j=1}^{N_{v i}} u_{j} \phi_{j}+\sum_{j=N_{v i}+1}^{N_{v}} u_{j} \phi_{j},
\end{gathered}
$$

where $\phi_{j}$ is the linear basis function associated with the $j$-th vertex, $\boldsymbol{a}_{j}$. The boundary condition (2) is approximated by

$$
u_{j}=g\left(\boldsymbol{a}_{j}\right), \quad j=N_{v i}+1, \ldots, N_{v} .
$$

Substituting (9) into and taking $v^{h}=\phi_{i}\left(i=1, \ldots, N_{v i}\right)$ in (7) and combining the resulting equations with 10 , we obtain the linear algebraic system

$$
A \boldsymbol{u}=\boldsymbol{f}
$$

where

$$
A=\left[\begin{array}{cc}
A_{11} & A_{12} \\
0 & I
\end{array}\right]
$$




$$
\begin{gathered}
\boldsymbol{u}=\left(u_{1}, \ldots, u_{N_{v i}}, u_{N_{v i}+1}, \ldots, u_{N_{v}}\right)^{T}, \\
\boldsymbol{f}=\left(f_{1}, \ldots, f_{N_{v i}}, g_{N_{v i}+1}, \ldots, g_{N_{v}}\right)^{T},
\end{gathered}
$$

and $I$ in $\sqrt{12}$ is the identity matrix of size $\left(N_{v}-N_{v i}\right)$. The entries of the stiffness matrix $A$ and the right-hand-side vector $\boldsymbol{f}$ are given by

$$
\begin{gathered}
a_{i j}=\left.\sum_{K \in \mathcal{T}_{h}}|K|\left(\left.\nabla \phi_{i}\right|_{K}\right)^{T} \mathbb{D}_{K} \nabla \phi_{j}\right|_{K}, \quad i=1, \ldots, N_{v i}, j=1, \ldots, N_{v} \\
f_{i}=\sum_{K \in \mathcal{T}_{h}}|K| \sum_{k=1}^{m} \hat{w}_{k} f\left(b_{k}^{K}\right) \phi_{i}\left(b_{k}^{K}\right), \quad i=1, \ldots, N_{v i} .
\end{gathered}
$$

The expression (13) can be simplified. Let $\omega_{i}$ be the patch of the elements sharing vertex $\boldsymbol{a}_{i}$. Noticing that $\nabla \phi_{i}=0$ for $(x, y) \notin \omega_{i}$, we have, for $i \neq j, i=1, \ldots, N_{v i}, j=1, \ldots, N_{v}$,

$$
\begin{aligned}
a_{i j} & =\left.\sum_{K \in \omega_{i} \cap \omega_{j}}|K|\left(\left.\nabla \phi_{i}\right|_{K}\right)^{T} \mathbb{D}_{K} \nabla \phi_{j}\right|_{K} \\
& =\left.|K|\left(\left.\nabla \phi_{i}\right|_{K}\right)^{T} \mathbb{D}_{K} \nabla \phi_{j}\right|_{K}+\left.\left|K^{\prime}\right|\left(\left.\nabla \phi_{i}\right|_{K^{\prime}}\right)^{T} \mathbb{D}_{K^{\prime}} \nabla \phi_{j}\right|_{K^{\prime}} .
\end{aligned}
$$

In (15), $K$ and $K^{\prime}$ denote the two elements sharing the common edge $\left(e_{i j}\right)$ connecting vertices $\boldsymbol{a}_{i} \equiv \boldsymbol{a}_{i}^{K} \equiv \boldsymbol{a}_{i}^{K^{\prime}}$ and $\boldsymbol{a}_{j} \equiv \boldsymbol{a}_{j}^{K} \equiv \boldsymbol{a}_{j}^{K^{\prime}}$; see Fig. 1,

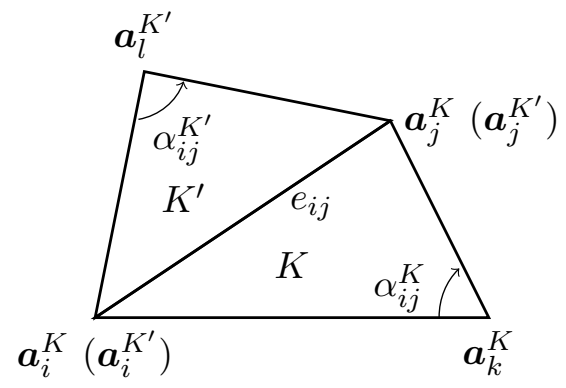

Figure 1: Elements $K$ and $K^{\prime}$ share the common edge $\left(e_{i j}\right)$ connecting vertices $\boldsymbol{a}_{i}^{K}\left(\boldsymbol{a}_{i}^{K^{\prime}}\right)$ and $\boldsymbol{a}_{j}^{K}$ $\left(\boldsymbol{a}_{j}^{K^{\prime}}\right)$. The angles opposite the edge are denoted by $\alpha_{i j}^{K}$ and $\alpha_{i j}^{K^{\prime}}$, respectively. The Delaunay condition is $\alpha_{i j}^{K}+\alpha_{i j}^{K^{\prime}} \leq \pi$.

\section{The anisotropic non-obtuse angle condition}

In this section, we study mesh conditions under which the linear finite element scheme (7) satisfies DMP.

To start with, we introduce some notation. Denote the vertices of an element $K$ by $\boldsymbol{a}_{1}^{K}, \boldsymbol{a}_{2}^{K}, \boldsymbol{a}_{3}^{K}$. The edge matrix of $K$ is defined as

$$
E_{K}=\left[\boldsymbol{a}_{2}^{K}-\boldsymbol{a}_{1}^{K}, \boldsymbol{a}_{3}^{K}-\boldsymbol{a}_{1}^{K}\right] .
$$

Since $K$ is simplicial, $E_{K}$ is nonsingular [37]. A set of $\boldsymbol{q}$-vectors (cf. Fig. 2) can then be defined as

$$
\left[\boldsymbol{q}_{2}^{K}, \boldsymbol{q}_{3}^{K}\right]=E_{K}^{-T}, \quad \boldsymbol{q}_{1}^{K}=-\boldsymbol{q}_{2}^{K}-\boldsymbol{q}_{3}^{K} .
$$




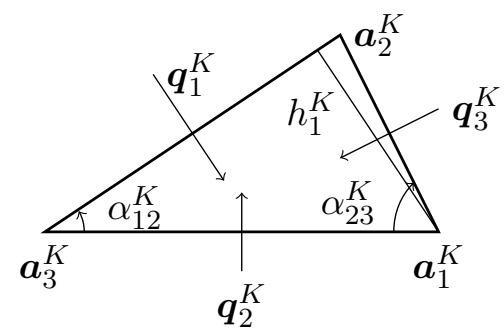

Figure 2: A sketch of the $\boldsymbol{q}$ vectors and other geometric quantities for an arbitrary element $K$.

By definition, $\boldsymbol{q}_{i}^{K}$ is the inward normal to the edge opposite to vertex $\boldsymbol{a}_{i}^{K}$ (i.e., the edge not having $\boldsymbol{a}_{i}^{K}$ as a vertex). This orthogonality implies that the (dihedral) angle, $\alpha_{i j}^{K}$, opposite to edge $e_{i j}$ can be calculated in terms of $\boldsymbol{q}_{i}^{K}$ and $\boldsymbol{q}_{j}^{K}$ as

$$
\alpha_{i j}^{K}=\pi-\arccos \left(\frac{\boldsymbol{q}_{i}^{K} \cdot \boldsymbol{q}_{j}^{K}}{\left\|\boldsymbol{q}_{i}^{K}\right\| \cdot\left\|\boldsymbol{q}_{j}^{K}\right\|}\right), \quad i \neq j .
$$

Moreover, it is known [3, 23] that

$$
\left.\nabla \phi_{i}\right|_{K}=\boldsymbol{q}_{i}^{K}
$$

From this relation, it is not difficult to show

$$
\left\|\boldsymbol{q}_{i}^{K}\right\|=\frac{1}{h_{i}^{K}}
$$

where $h_{i}^{K}$ is the height of $K$ in the direction of $\boldsymbol{q}_{i}^{K}$ or the shortest distance from $\boldsymbol{a}_{i}^{K}$ to the edge opposite to $\boldsymbol{a}_{i}^{K}$; see Fig. 2 .

Now, we are ready to describe the anisotropic non-obtuse angle condition.

Lemma 3.1 If the mesh satisfies the anisotropic non-obtuse angle condition

$$
\left(\boldsymbol{q}_{i}^{K}\right)^{T} \mathbb{D}_{K} \boldsymbol{q}_{j}^{K} \leq 0, \quad \forall i \neq j, i, j=1,2,3, \forall K \in \mathcal{T}_{h}
$$

then the linear finite element scheme (7) for solving BVP (1) and (2) satisfies DMP.

This lemma was proven in 28] in any spatial dimension by showing that the stiffness matrix $A$ in (11) is an $M$-matrix and has non-negative row sums. A key step of the proof is to show $a_{i j} \leq 0$ for all $i \neq j$, which can be seen to hold from (15), (18), and 20).

For the isotropic diffusion case, the condition 20 reduces to

$$
\left(\boldsymbol{q}_{i}^{K}\right)^{T} \boldsymbol{q}_{j}^{K} \leq 0, \quad \forall i \neq j, i, j=1,2,3, \forall K \in \mathcal{T}_{h}
$$

Thus, 20 is a generalization of 21) for a general diffusion matrix. Notice that 21) implies that the second angle on the right-hand side of (17) is between $\pi / 2$ and $\pi$. Consequently, (21) is exactly the non-obtuse angle condition [9], implying $\alpha_{i j}^{K} \leq \pi / 2$.

The condition (20) can be more directly interpreted as requiring the angles of elements to be non-obtuse when measured in a metric depending on $\mathbb{D}$. To see this, we first notice that, according 
to 20 , the angle between $\boldsymbol{q}_{i}^{K}$ and $\boldsymbol{q}_{j}^{K}$ should be measured in the metric $\mathbb{D}_{K}$. Indeed, it has the expression

$$
\arccos \left(\frac{\left(\boldsymbol{q}_{i}^{K}\right)^{T} \mathbb{D}_{K} \boldsymbol{q}_{j}^{K}}{\left\|\boldsymbol{q}_{i}^{K}\right\|_{\mathbb{D}_{K}}\left\|\boldsymbol{q}_{j}^{K}\right\|_{\mathbb{D}_{K}}}\right)
$$

where the $\mathbb{D}_{K}$-norm is defined by

$$
\|\boldsymbol{v}\|_{\mathbb{D}_{K}}=\sqrt{\boldsymbol{v}^{T} \mathbb{D}_{K} \boldsymbol{v}}, \quad \forall \boldsymbol{v} \in \mathbb{R}^{2} .
$$

Since

$$
\arccos \left(\frac{\left(\boldsymbol{q}_{i}^{K}\right)^{T} \mathbb{D}_{K} \boldsymbol{q}_{j}^{K}}{\left\|\boldsymbol{q}_{i}^{K}\right\|_{\mathbb{D}_{K}}\left\|\boldsymbol{q}_{j}^{K}\right\|_{\mathbb{D}_{K}}}\right)=\arccos \left(\frac{\left(\mathbb{D}_{K}^{\frac{1}{2}} \boldsymbol{q}_{i}^{K}\right)^{T}\left(\mathbb{D}_{K}^{\frac{1}{2}} \boldsymbol{q}_{j}^{K}\right)}{\left\|\mathbb{D}_{K}^{\frac{1}{2}} \boldsymbol{q}_{i}^{K}\right\| \cdot\left\|\mathbb{D}_{K}^{\frac{1}{2}} \boldsymbol{q}_{j}^{K}\right\|}\right)
$$

the angle can also be regarded as the one between vectors $\mathbb{D}_{K}^{\frac{1}{2}} \boldsymbol{q}_{i}^{K}$ and $\mathbb{D}_{K}^{\frac{1}{2}} \boldsymbol{q}_{j}^{K}$ in the Euclidean norm. Denote the third vertex of $K$ by $\boldsymbol{a}_{k}^{K}$. By definition, $\boldsymbol{q}_{i}^{K}$ and $\boldsymbol{q}_{j}^{K}$ are orthogonal to edges $\left(\boldsymbol{a}_{j}^{K}-\boldsymbol{a}_{k}^{K}\right)$ and $\left(\boldsymbol{a}_{i}^{K}-\boldsymbol{a}_{k}^{K}\right)$, respectively; i.e.,

$$
\left(\boldsymbol{q}_{i}^{K}\right)^{T}\left(\boldsymbol{a}_{j}^{K}-\boldsymbol{a}_{k}^{K}\right)=0, \quad\left(\boldsymbol{q}_{j}^{K}\right)^{T}\left(\boldsymbol{a}_{i}^{K}-\boldsymbol{a}_{k}^{K}\right)=0 .
$$

It follows that

$$
\left(\mathbb{D}_{K}^{\frac{1}{2}} \boldsymbol{q}_{i}^{K}\right)^{T}\left(\mathbb{D}_{K}^{-\frac{1}{2}}\left(\boldsymbol{a}_{j}^{K}-\boldsymbol{a}_{k}^{K}\right)\right)=0, \quad\left(\mathbb{D}_{K}^{\frac{1}{2}} \boldsymbol{q}_{j}^{K}\right)^{T}\left(\mathbb{D}_{K}^{-\frac{1}{2}}\left(\boldsymbol{a}_{i}^{K}-\boldsymbol{a}_{k}^{K}\right)\right)=0,
$$

indicating that $\mathbb{D}_{K}^{\frac{1}{2}} \boldsymbol{q}_{i}^{K}$ and $\mathbb{D}_{K}^{\frac{1}{2}} \boldsymbol{q}_{j}^{K}$ are orthogonal to $\mathbb{D}_{K}^{-\frac{1}{2}}\left(\boldsymbol{a}_{j}^{K}-\boldsymbol{a}_{k}^{K}\right)$ and $\mathbb{D}_{K}^{-\frac{1}{2}}\left(\boldsymbol{a}_{i}^{K}-\boldsymbol{a}_{k}^{K}\right)$, respectively. Thus, the angle between edges $\left(\boldsymbol{a}_{j}^{K}-\boldsymbol{a}_{k}^{K}\right)$ and $\left(\boldsymbol{a}_{i}^{K}-\boldsymbol{a}_{k}^{K}\right)$ in the $\mathbb{D}_{K}^{-1}$-norm and that between $\boldsymbol{q}_{i}^{K}$ and $\boldsymbol{q}_{j}^{K}$ in the $\mathbb{D}_{K}$ norm are related by

$$
\arccos \left(\frac{\left(\boldsymbol{q}_{i}^{K}\right)^{T} \mathbb{D}_{K} \boldsymbol{q}_{j}^{K}}{\left\|\boldsymbol{q}_{i}^{K}\right\|_{\mathbb{D}_{K}}\left\|\boldsymbol{q}_{j}^{K}\right\|_{\mathbb{D}_{K}}}\right)+\arccos \left(\frac{\left(\boldsymbol{a}_{i}^{K}-\boldsymbol{a}_{k}^{K}\right)^{T} \mathbb{D}_{K}^{-1}\left(\boldsymbol{a}_{j}^{K}-\boldsymbol{a}_{k}^{K}\right)}{\left\|\left(\boldsymbol{a}_{i}^{K}-\boldsymbol{a}_{k}^{K}\right)\right\|_{\mathbb{D}_{K}^{-1}}\left\|\left(\boldsymbol{a}_{j}^{K}-\boldsymbol{a}_{k}^{K}\right)\right\|_{\mathbb{D}_{K}^{-1}}}\right)=\pi .
$$

Since (20) means the first angle on the left-hand side of the above equation is between $\pi / 2$ and $\pi$, we conclude that condition (20) is equivalent to the requirement that the angles of elements be non-obtuse when measured in the $\mathbb{D}_{K}^{-1}$ norm.

It should be emphasized that condition (20) has been obtained by considering only local stiffness matrices on individual elements. For the current 2D situation, this means that each term in (15) has been required to be non-positive. Clearly, this is too strong since we only need $a_{i j} \leq 0$ for $i \neq j$ for $A$ to be an $M$-matrix. For the special case $\mathbb{D}=I$, the Delaunay condition requiring the sum of any pair of angles opposite a common edge to be less than or equal to $\pi$ (cf. Fig. 1) is sufficient to guarantee $a_{i j} \leq 0$ for $i \neq j$. It is then natural to ask if condition (20) can be weakened and a Delaunay-type condition exists for the general diffusion matrix $\mathbb{D}$. This issue is studied in the next section.

\section{A Delaunay-type mesh condition}

In this section, we develop a Delaunay-type mesh condition under which the linear finite element scheme (7) satisfies DMP. The main result is given in Theorem 4.1. Its proof is broken into a series of Lemmas. 
Lemma 4.1 For any element $K$,

$$
\left.|K|\left(\left.\nabla \phi_{i}\right|_{K}\right)^{T} \nabla \phi_{j}\right|_{K}=-\frac{1}{2} \cot \left(\alpha_{i j}^{K}\right), \quad i \neq j, i, j=1,2,3
$$

where $\alpha_{i j}^{K}$ is the angle between edges $e_{k i}$ and $e_{k j}$, with $\boldsymbol{a}_{k}^{K}$ being the third vertex.

Proof. This result has been obtained in [14]. For completeness, we give a short proof here. Without loss of generality, we consider the case with $i=1, j=2$, and $k=3$ (cf. Fig. 2). From (17), (18), and (19), we have

$$
\begin{aligned}
\left.|K|\left(\left.\nabla \phi_{1}\right|_{K}\right)^{T} \nabla \phi_{2}\right|_{K} & =|K|\left(\boldsymbol{q}_{1}^{K}\right)^{T} \boldsymbol{q}_{2}^{K} \\
& =|K|\left\|\boldsymbol{q}_{1}^{K}\right\| \cdot\left\|\boldsymbol{q}_{2}^{K}\right\| \cos \left(\pi-\alpha_{12}^{K}\right) \\
& =-\frac{|K|}{h_{1}^{K} h_{2}^{K}} \cos \left(\alpha_{12}^{K}\right) .
\end{aligned}
$$

From Fig. 2, it is easy to see

$$
|K|=\frac{1}{2} h_{2}^{K}\left\|\boldsymbol{a}_{1}^{K}-\boldsymbol{a}_{3}^{K}\right\|=\frac{h_{1}^{K} h_{2}^{K}}{2 \sin \left(\alpha_{12}^{K}\right)} .
$$

Combining the above results, we obtain inequality (24).

The angle $\alpha_{i j}^{K}$ can be calculated in terms of the $\boldsymbol{q}$ vectors as in 17 or in terms of the edge vectors as

$$
\alpha_{i j}^{K}=\arccos \left(\frac{\left(\boldsymbol{a}_{i}^{K}-\boldsymbol{a}_{k}^{K}\right)^{T}\left(\boldsymbol{a}_{j}^{K}-\boldsymbol{a}_{k}^{K}\right)}{\left\|\boldsymbol{a}_{i}^{K}-\boldsymbol{a}_{k}^{K}\right\|\left\|\boldsymbol{a}_{j}^{K}-\boldsymbol{a}_{k}^{K}\right\|}\right) .
$$

The above formula is more desirable if linear coordinate transformations are involved. This is because, under a linear coordinate transformation, the edge vectors of $K$ will remain to be the edge vectors of the transformed element but in general the $\boldsymbol{q}$ vectors will not. The latter is due to the fact that orthogonality between vectors is not preserved by linear coordinate transformations.

Lemma 4.2 For any element $K$,

$$
\left.|K|\left(\left.\nabla \phi_{i}\right|_{K}\right)^{T} \mathbb{D}_{K} \nabla \phi_{j}\right|_{K}=-\frac{\sqrt{\operatorname{det}\left(\mathbb{D}_{K}\right)}}{2} \cot \left(\alpha_{i j, \mathbb{D}_{K}^{-1}}^{K}\right), \quad i \neq j, i, j=1,2,3
$$

where $\alpha_{i j, \mathbb{D}_{K}^{-1}}^{K}$ is the angle between edges $e_{k i}$ and $e_{k j}$ (with $\boldsymbol{a}_{k}^{K}$ being the third vertex) measured in the metric $\mathbb{D}_{K}^{-1}$, i.e.,

$$
\alpha_{i j, \mathbb{D}_{K}^{-1}}^{K}=\arccos \left(\frac{\left(\boldsymbol{a}_{i}^{K}-\boldsymbol{a}_{k}^{K}\right)^{T} \mathbb{D}_{K}^{-1}\left(\boldsymbol{a}_{j}^{K}-\boldsymbol{a}_{k}^{K}\right)}{\left\|\boldsymbol{a}_{i}^{K}-\boldsymbol{a}_{k}^{K}\right\|_{\mathbb{D}_{K}^{-1}}\left\|\boldsymbol{a}_{j}^{K}-\boldsymbol{a}_{k}^{K}\right\|_{\mathbb{D}_{K}^{-1}}}\right) .
$$

Proof. Consider a linear mapping $G: K \rightarrow \tilde{K}$ defined as

$$
\left(\begin{array}{l}
\xi \\
\eta
\end{array}\right)=\mathbb{D}_{K}^{-\frac{1}{2}}\left(\begin{array}{l}
x \\
y
\end{array}\right), \quad \forall(x, y) \in K
$$


where $\tilde{K}=G(K)$ and $(x, y)$ and $(\xi, \eta)$ are the coordinates in $K$ and $\tilde{K}$, respectively. Let $\tilde{\boldsymbol{a}}_{i}=G\left(\boldsymbol{a}_{i}^{K}\right)$ $(i=1,2,3), \tilde{e}_{i j}=G\left(e_{i j}\right)(i \neq j)$, and $\tilde{\nabla}=((\partial / \partial \xi),(\partial / \partial \eta))^{T}$. Denote the angles of $\tilde{K}$ by $\tilde{\alpha}_{i j}$. It is easy to show that $\tilde{e}_{i j}$ 's form the edges of $\tilde{K}$ and $\left.\tilde{\phi}_{i}(\xi, \eta) \equiv \phi_{i}\right|_{K}\left(F^{-1}(\xi, \eta)\right)(i=1,2,3)$ form the linear basis functions on $\tilde{K}$. Moreover,

$$
\nabla=\mathbb{D}_{K}^{-\frac{1}{2}} \tilde{\nabla}
$$

Since $\nabla \phi_{i}$ and $\nabla \phi_{j}$ are constant on $K$, we have

$$
\begin{aligned}
\left.|K|\left(\left.\nabla \phi_{i}\right|_{K}\right)^{T} \mathbb{D}_{K} \nabla \phi_{j}\right|_{K} & =\int_{K}\left(\nabla \phi_{i}\right)^{T} \mathbb{D}_{K} \nabla \phi_{j} d x d y \\
& =\int_{\tilde{K}}\left(\tilde{\nabla} \tilde{\phi}_{i}\right)^{T} \tilde{\nabla} \tilde{\phi}_{j} \operatorname{det}\left(\mathbb{D}_{K}^{\frac{1}{2}}\right) d \xi d \eta \\
& =\left.\sqrt{\operatorname{det}\left(\mathbb{D}_{K}\right)}|\tilde{K}|\left(\left.\tilde{\nabla} \tilde{\phi}_{i}\right|_{\tilde{K}}\right)^{T} \tilde{\nabla}_{j}\right|_{\tilde{K}}
\end{aligned}
$$

Applying Lemma 4.1 to the last term in the above equation on element $\tilde{K}$, we have

$$
\left.|K|\left(\left.\nabla \phi_{i}\right|_{K}\right)^{T} \mathbb{D}_{K} \nabla \phi_{j}\right|_{K}=-\frac{\sqrt{\operatorname{det}\left(\mathbb{D}_{K}\right)}}{2} \cot \left(\tilde{\alpha}_{i j}\right) .
$$

From

$$
\tilde{\boldsymbol{a}}_{i}-\tilde{\boldsymbol{a}}_{k}=\mathbb{D}_{K}^{-\frac{1}{2}}\left(\boldsymbol{a}_{i}^{K}-\boldsymbol{a}_{k}^{K}\right), \quad\left\|\tilde{\boldsymbol{a}}_{i}-\tilde{\boldsymbol{a}}_{k}\right\|=\left\|\boldsymbol{a}_{i}^{K}-\boldsymbol{a}_{k}^{K}\right\|_{\mathbb{D}_{K}^{-1}}
$$

and similar formulas for $\left(\boldsymbol{a}_{j}^{K}-\boldsymbol{a}_{k}^{K}\right), \tilde{\alpha}_{i j}$ can be expressed as

$$
\begin{aligned}
\tilde{\alpha}_{i j} & =\arccos \left(\frac{\left(\tilde{\boldsymbol{a}}_{i}-\tilde{\boldsymbol{a}}_{k}\right)^{T}\left(\tilde{\boldsymbol{a}}_{j}-\tilde{\boldsymbol{a}}_{k}\right)}{\left\|\tilde{\boldsymbol{a}}_{i}-\tilde{\boldsymbol{a}}_{k}\right\|\left\|\tilde{\boldsymbol{a}}_{j}-\tilde{\boldsymbol{a}}_{k}\right\|}\right) \\
& =\arccos \left(\frac{\left(\boldsymbol{a}_{i}^{K}-\boldsymbol{a}_{k}^{K}\right)^{T} \mathbb{D}_{K}^{-1}\left(\boldsymbol{a}_{j}^{K}-\boldsymbol{a}_{k}^{K}\right)}{\left\|\boldsymbol{a}_{i}^{K}-\boldsymbol{a}_{k}^{K}\right\|_{\mathbb{D}_{K}^{-1}}\left\|\boldsymbol{a}_{j}^{K}-\boldsymbol{a}_{k}^{K}\right\|_{\mathbb{D}_{K}^{-1}}}\right) \\
& =\alpha_{i j, \mathbb{D}_{K}^{-1}}^{K} .
\end{aligned}
$$

Combining this result with 29 gives 26 .

Lemma 4.3 The entry $a_{i j}$ of the stiffness matrix A, 15), can be expressed as

$$
a_{i j}=-\frac{\sqrt{\operatorname{det}\left(\mathbb{D}_{K}\right)}}{2} \cot \left(\alpha_{i j, \mathbb{D}_{K}^{-1}}^{K}\right)-\frac{\sqrt{\operatorname{det}\left(\mathbb{D}_{K^{\prime}}\right)}}{2} \cot \left(\alpha_{i j, \mathbb{D}_{K^{\prime}}^{-1}}^{K^{\prime}}\right) .
$$

Proof. This lemma is a consequence of combination of $(15)$ and Lemma 4.2 .

Theorem 4.1 If the triangular mesh satisfies

$$
\begin{aligned}
& \frac{1}{2}\left[\alpha_{i j, \mathbb{D}_{K}^{-1}}^{K}+\alpha_{i j, \mathbb{D}_{K^{\prime}}^{-1}}^{K^{\prime}}+\operatorname{arccot}\left(\sqrt{\frac{\operatorname{det}\left(\mathbb{D}_{K}\right)}{\operatorname{det}\left(\mathbb{D}_{K^{\prime}}\right)}} \cot \left(\alpha_{i j, \mathbb{D}_{K}^{-1}}^{K}\right)\right)\right. \\
& +\operatorname{arccot}\left(\sqrt{\frac{\operatorname{det}\left(\mathbb{D}_{K^{\prime}}\right)}{\operatorname{det}\left(\mathbb{D}_{K}\right)}} \cot \left(\alpha_{i j, \mathbb{D}_{K^{\prime}}^{-1}}^{K^{\prime}}\right)\right] \leq \pi, \quad \text { for all interior edges } e_{i j}
\end{aligned}
$$

where $K$ and $K^{\prime}$ are the elements sharing $e_{i j}$, then the linear finite element scheme (7) satisfies $D M P$. 
Proof. We first show that if the mesh satisfies

$$
\alpha_{i j, \mathbb{D}_{K}^{-1}}^{K}+\operatorname{arccot}\left(\sqrt{\frac{\operatorname{det}\left(\mathbb{D}_{K^{\prime}}\right)}{\operatorname{det}\left(\mathbb{D}_{K}\right)}} \cot \left(\alpha_{i j, \mathbb{D}_{K^{\prime}}^{-1}}^{K^{\prime}}\right) \leq \pi, \quad \text { for all interior edges } e_{i j}\right.
$$

then the conclusion holds. Indeed, notice that the inequality

$$
\frac{\sqrt{\operatorname{det}\left(\mathbb{D}_{K}\right)}}{2} \cot \left(\alpha_{i j, \mathbb{D}_{K}^{-1}}^{K}\right)+\frac{\sqrt{\operatorname{det}\left(\mathbb{D}_{K^{\prime}}\right)}}{2} \cot \left(\alpha_{i j, \mathbb{D}_{K^{\prime}}^{-1}}^{K^{\prime}}\right) \geq 0
$$

can be written as

$$
\alpha_{i j, \mathbb{D}_{K}^{-1}}^{K} \leq \operatorname{arccot}\left(-\sqrt{\frac{\operatorname{det}\left(\mathbb{D}_{K^{\prime}}\right)}{\operatorname{det}\left(\mathbb{D}_{K}\right)}} \cot \left(\alpha_{i j, \mathbb{D}_{K^{\prime}}^{-1}}^{K^{\prime}}\right)\right)=\pi-\operatorname{arccot}\left(\sqrt{\frac{\operatorname{det}\left(\mathbb{D}_{K^{\prime}}\right)}{\operatorname{det}\left(\mathbb{D}_{K}\right)}} \cot \left(\alpha_{\left.i j, \mathbb{D}_{K^{\prime}}^{-1}\right)}^{K^{\prime}}\right),\right.
$$

which is exactly $(32)$. Then, from Lemma 4.3 we have $a_{i j} \leq 0$ for $i=1, \ldots, N_{v i}$ and $j=1, \ldots, N_{v}$ if (32) is satisfied. The result also means $a_{i j} \leq 0$ for all $i \neq j$ due to the special structure (12) of the stiffness matrix. Following the proof of Theorem 2.1 of [28] we can then show that $A$ is an $M$-matrix and has non-negative row sums, which implies that the linear finite element scheme (7) satisfies DMP (cf. Stoyan [40] or Lemma 1.2 of [28]).

Next, it is easy to show that $(32)$ is equivalent to

$$
\alpha_{i j, \mathbb{D}_{K^{\prime}}^{-1}}^{K^{\prime}}+\operatorname{arccot}\left(\sqrt{\frac{\operatorname{det}\left(\mathbb{D}_{K}\right)}{\operatorname{det}\left(\mathbb{D}_{K^{\prime}}\right)}} \cot \left(\alpha_{i j, \mathbb{D}_{K}^{-1}}^{K}\right)\right) \leq \pi
$$

As a result, (31) and (32) are mathematically equivalent.

We now study the mesh condition (31). We first consider the case with constant $\mathbb{D}$. For this case,

$$
\mathbb{D}_{K}=\mathbb{D}_{K^{\prime}}=\mathbb{D}, \quad \operatorname{det}\left(\mathbb{D}_{K}\right)=\operatorname{det}\left(\mathbb{D}_{K^{\prime}}\right)=\operatorname{det}(\mathbb{D})
$$

Then (31) reduces to

$$
\alpha_{i j, \mathbb{D}^{-1}}^{K}+\alpha_{i j, \mathbb{D}^{-1}}^{K^{\prime}} \leq \pi, \quad \text { for all interior edges } e_{i j} .
$$

For the special case with $\mathbb{D}=I$, (34) reduces to

$$
\alpha_{i j}^{K}+\alpha_{i j}^{K^{\prime}} \leq \pi, \quad \text { for all interior edges } e_{i j}
$$

which is exactly the Delaunay condition (cf. Fig. 1). Thus, the mesh condition (31) reduces to the Delaunay condition for the special case $\mathbb{D}=I$ and is a generalization of the Delaunay condition for a general $\mathbb{D}$.

Next, we consider the mesh condition

$$
\alpha_{i j, \mathbb{D}_{K}^{-1}}^{K} \leq \frac{\pi}{2}, \quad i \neq j, i, j=1,2,3, \quad \forall K \in \mathcal{T}_{h}
$$

for a general matrix-valued function $\mathbb{D}=\mathbb{D}(x, y)$. From 23) and 27) it is not difficult to see that this mesh condition is equivalent to the anisotropic non-obtuse angle condition (20). Moreover, under (36) we have

$$
\alpha_{i j, \mathbb{D}_{K}^{-1}}^{K} \leq \frac{\pi}{2}, \quad \cot \left(\alpha_{i j, \mathbb{D}_{K}^{-1}}^{K}\right) \geq 0, \quad \operatorname{arccot}\left(\sqrt{\frac{\operatorname{det}\left(\mathbb{D}_{K}\right)}{\operatorname{det}\left(\mathbb{D}_{K^{\prime}}\right)}} \cot \left(\alpha_{i j, \mathbb{D}_{K}^{-1}}^{K}\right)\right) \leq \frac{\pi}{2},
$$


and similar results for $\alpha_{i j, \mathbb{D}_{K^{\prime}}^{-1}}^{K^{\prime}}$ and thus $\sqrt{31}$ is true. Therefore, $\sqrt{36}$, or equivalently $\sqrt{20}$, implies (31). In other words, the mesh condition (31) is weaker than the anisotropic non-obtuse angle condition (20).

Finally, we consider some special cases for (31). It is obvious that (31) reduces to (34) when $\operatorname{det}\left(\mathbb{D}_{K}\right)=\operatorname{det}\left(\mathbb{D}_{K^{\prime}}\right)$. In Fig. 3 the region of $\left(\alpha_{i j, \mathbb{D}_{K^{\prime}}^{K^{\prime}}}, \alpha_{i j, \mathbb{D}_{K}^{-1}}^{K}\right)$ satisfying the mesh condition 31 is plotted for two cases where the ratio $\operatorname{det}\left(\mathbb{D}_{K^{\prime}}\right) / \operatorname{det}\left(\mathbb{D}_{K}\right)$ is either large or small. From the figure, one can see that when the ratio is large (Fig. $3(\mathrm{a})$ ), $\alpha_{i j, \mathbb{D}_{K^{\prime}}^{-1}}^{K^{\prime}}$ should essentially be non-obtuse whereas $\alpha_{i j, \mathbb{D}_{K}^{-1}}^{K}$ can basically be any angle between 0 and $\pi$. On the other hand, when the ratio is small (Fig. $3(\mathrm{~b})$ ), the roles of $\alpha_{i j, \mathbb{D}_{K}^{-1}}^{K}$ and $\alpha_{i j, \mathbb{D}_{K^{\prime}}^{-1}}^{K^{\prime}}$ switch. This observation is consistent with that made by $\mathrm{Xu}$ and Zikatanov [44] that the non-obtuse angle condition should be imposed at edges where the diffusion coefficient is discontinuous (and thus the ratio $\operatorname{det}\left(\mathbb{D}_{K^{\prime}}\right) / \operatorname{det}\left(\mathbb{D}_{K}\right)$ can be large or small) to guarantee DMP satisfaction. It is also interesting to observe from Fig. 3 that the DMP satisfaction region overlaps with $\alpha_{i j, \mathbb{D}_{K}^{-1}}^{K}+\alpha_{i j, \mathbb{D}_{K^{\prime}}^{-1}}^{K^{\prime}} \geq \pi$.
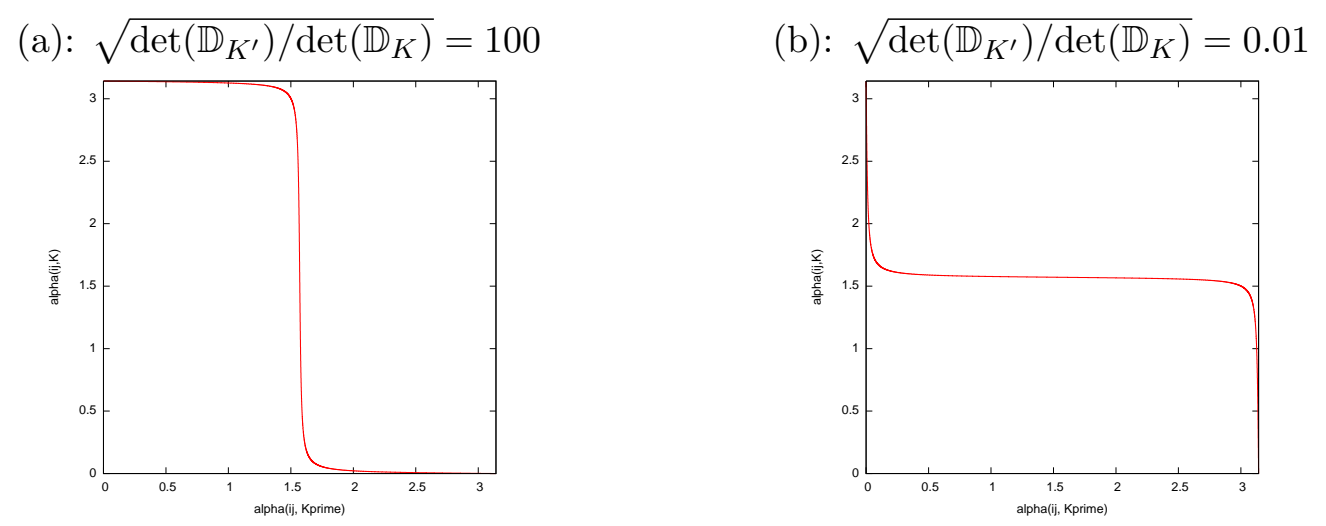

Figure 3: The $x$ and $y$ axes are $\alpha_{i j, \mathbb{D}_{K^{\prime}}^{-1}}^{K^{\prime}}$ and $\alpha_{i j, \mathbb{D}_{K}^{-1}}^{K}$, respectively. The DMP satisfaction region (satisfying the mesh condition (31) ) is below the plotted curve.

\section{$5 \quad$ Numerical results}

In this section, we present some numerical results obtained for BVP (1) and (2) with

$$
\begin{gathered}
f \equiv 0, \quad g(x, 0)=g(16, y)=0, \\
g(0, y)=\left\{\begin{array}{ll}
0.5 y, & \text { for } 0 \leq y<2 \\
1, & \text { for } 14 \leq y \leq 16
\end{array} \text { and } g(x, 16)= \begin{cases}1, & \text { for } 0 \leq x \leq 14 \\
8-0.5 x, & \text { for } 14<x \leq 16\end{cases} \right.
\end{gathered}
$$

The diffusion matrix is defined as

$$
\mathbb{D}(x, y)=\left(\begin{array}{ll}
500.5 & 499.5 \\
499.5 & 500.5
\end{array}\right) .
$$

This example has a constant but anisotropic diffusion matrix $\mathbb{D}$ and a continuous boundary condition. It satisfies the maximum principle and its solution stays between 0 and 1. 
The computation is done with four types of triangular meshes shown in Fig. 4. Meshes (a) and (b) are obtained by dividing a rectangle into two triangles using the northwest diagonal line and the northeast line, respectively, Mesh (c) obtained by dividing a rectangle into four triangles with the intersection toward the northeast corner, and Mesh (d) is a Delaunay mesh (which satisfies the Delaunay condition). As mentioned in the previous section, mesh condition (31) reduces to (34) for the current example (with constant $\mathbb{D}$ ). Note that Meshes (a) and (d) do not satisfy (34) whereas Meshes (b) and (c) do (cf. Fig. 4). Especially, Mesh (c) has obtuse elements (with angles greater than $\pi / 2$ in the $\mathbb{D}^{-1}$-norm).

Fig. 5 shows the contours of the linear finite element solutions obtained for meshes finer than those shown in Fig. 4. One can see that finite element solutions for both Meshes (b) and (c) stay between 0 and 1 and show no undershoots and overshoots. This is consistent with Theorem 4.1. On the other hand, both Meshes (a) and (d) lead to undershoots and overshoots in the computational solutions. Fig. 6 shows these undershoots and overshoots as functions of the number of mesh elements. As the mesh is refined, the undershoots and overshoots decrease very slowly and eventually reach a rate $O\left(N^{-0.5}\right)$, where $N$ is the number of elements.

(a)

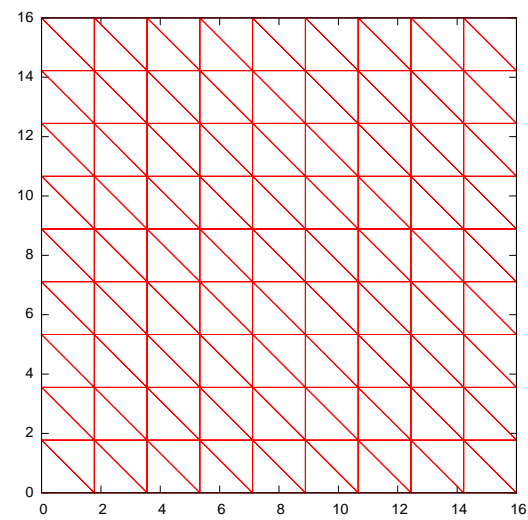

(c)

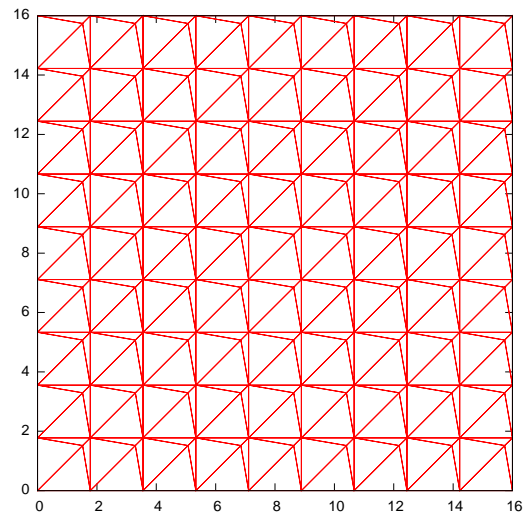

(b)

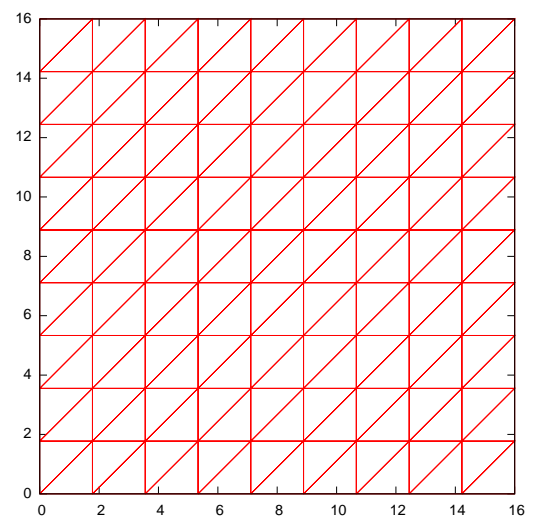

(d)

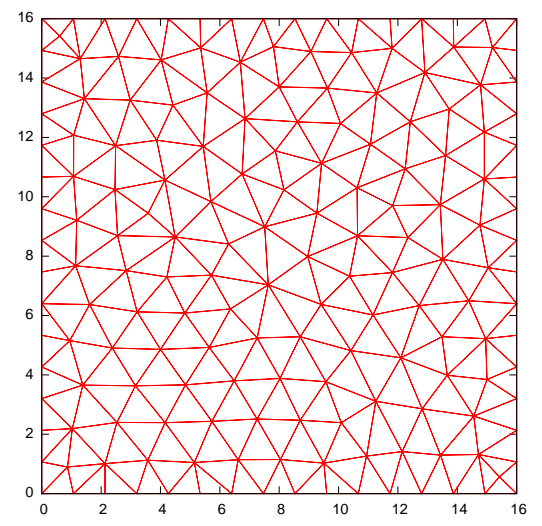

Figure 4: Numerical example in Section 5. Meshes used in computation. The maximum values for $\alpha_{i j, \mathbb{D}_{K}^{-1}}^{K}$ and $\left(\alpha_{i j, \mathbb{D}_{K}^{-1}}^{K}+\alpha_{i j, \mathbb{D}_{K^{\prime}}^{-1}}^{K^{\prime}}\right)$, respectively, are $0.98 \pi$ and $1.96 \pi$ for Mesh (a), $0.49 \pi$ and $0.98 \pi$ for Mesh (b), $0.51 \pi$ and $\pi$ for Mesh (c), and $0.98 \pi$ and $1.96 \pi$ for Mesh (d). 
(a)

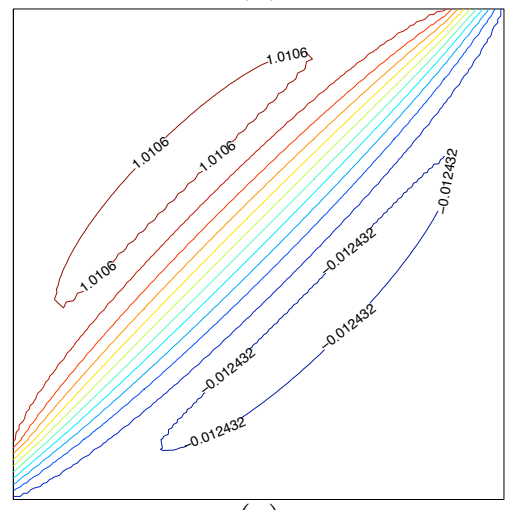

(c)

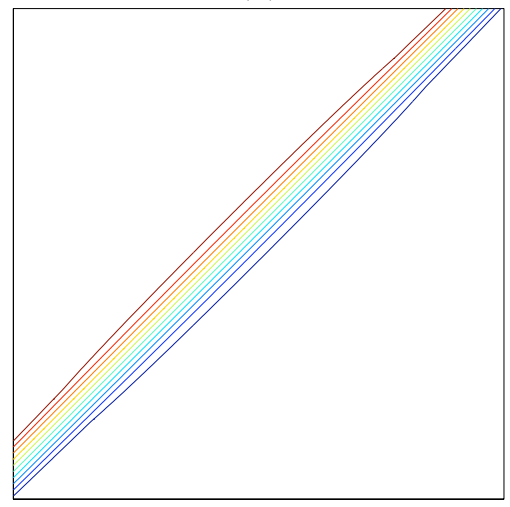

(b)

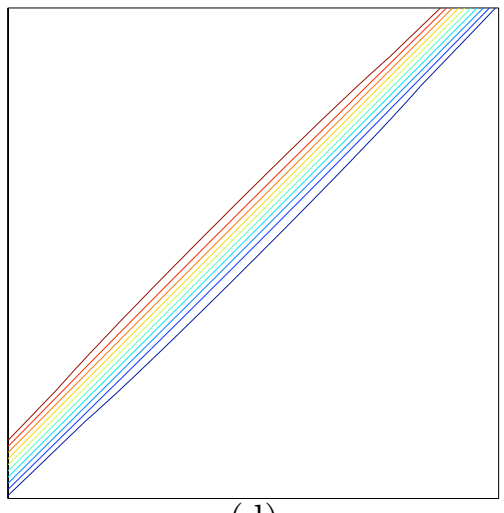

(d)

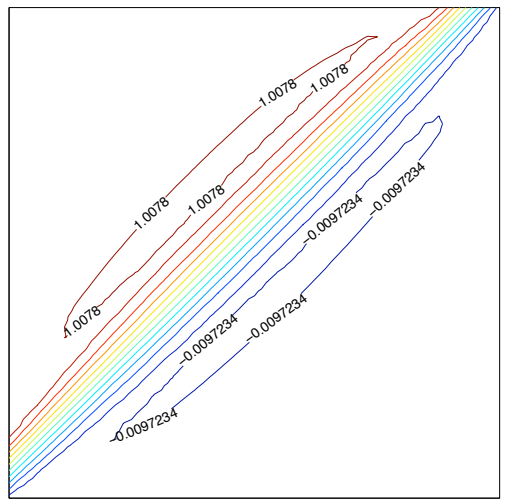

Figure 5: Numerical example in Section 5. Contours of linear finite element solutions.

\section{Conclusions and comments}

In the previous sections we have developed a Delaunay-type mesh condition (31) under which the linear finite element scheme (7) for solving the anisotropic diffusion problem (1) and (2) satisfies DMP. This condition is weaker than the anisotropic non-obtuse angle condition (20) developed in [28. It reduces to (34) when the diffusion matrix $\mathbb{D}$ is constant and especially to the Delaunay condition when $\mathbb{D}=I$. The main theoretical result is given in Theorem 4.1 and verified by numerical results.

It is well known that the Delaunay condition can be satisfied by a Delaunay mesh which can be generated through edge swapping from an existing triangular mesh. Moreover, Mlacnik and Durlofsky [32] have demonstrated that a properly designed edge swapping procedure can improve the monotonicity of finite volume approximations for anisotropic diffusion problems. Clearly, the mesh condition (31) can serve as a criterion for designing such a procedure. The development of an edge swapping procedure based on (31), the convergence study of edge swapping, and the generation of a mesh satisfying (31) through edge swapping may deserve future investigation. 
(a): For the type of mesh in Fig. 4(a).

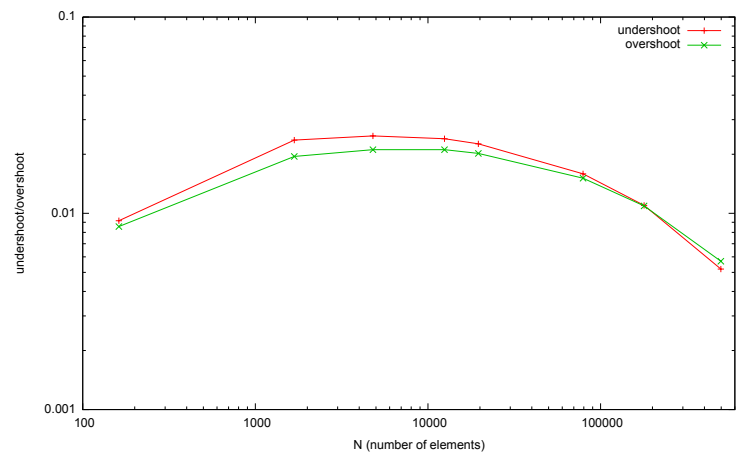

(b): For the type of mesh in Fig. 4(d).

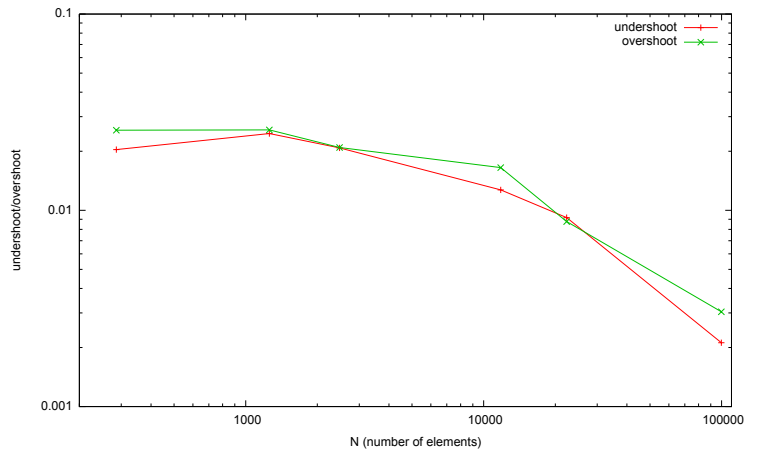

Figure 6: Numerical example in Section 5. Overshoots and undershoots as functions of the number of mesh elements.

\section{References}

[1] I. Aavatsmark, T. Barkve, Ø. Bøe, and T. Mannseth. Discretization on unstructured grids for inhomogeneous, anisotropic media. I. Derivation of the methods. SIAM J. Sci. Comput., 19:1700-1716 (electronic), 1998.

[2] I. Aavatsmark, T. Barkve, Ø. Bøe, and T. Mannseth. Discretization on unstructured grids for inhomogeneous, anisotropic media. II. Discussion and numerical results. SIAM J. Sci. Comput., 19:1717-1736 (electronic), 1998.

[3] J. Brandts, S. Korotov, and M. Křížek. Dissection of the path-simplex in $\mathbb{R}^{n}$ into $n$ pathsubsimplices. Lin. Alg. Appl., 421:382-393, 2007.

[4] J. Brandts, S. Korotov, and M. Kř́žzek. The discrete maximum principle for linear simplicial finite element approximations of a reaction-diffusion problem. Lin. Alg. Appl., 429:2344-2357, 2008 .

[5] E. Burman and A. Ern. Discrete maximum principle for Galerkin approximations of the Laplace operator on arbitrary meshes. C. R. Acad. Sci. Paris, Ser.I 338:641-646, 2004.

[6] T. F. Chan and J. Shen. Non-texture inpainting by curvature driven diffusions (CDD). J. Vis. Commun. Image Rep, 12:436-449, 2000.

[7] T. F. Chan, J. Shen, and L. Vese. Variational PDE models in image processing. Not. AMS J., 50:14-26, 2003.

[8] P. G. Ciarlet. Discrete maximum principle for finite difference operators. Aequationes Math., 4:338-352, 1970.

[9] P. G. Ciarlet and P.-A. Raviart. Maximum principle and uniform convergence for the finite element method. Comput. Meth. Appl. Mech. Eng., 2:17-31, 1973.

[10] P. I. Crumpton, G. J. Shaw, and A. F. Ware. Discretisation and multigrid solution of elliptic equations with mixed derivative terms and strongly discontinuous coefficients. J. Comput. Phys., 116:343-358, 1995. 
[11] A. Drăgănescu, T. F. Dupont, and L. R. Scott. Failure of the discrete maximum principle for an elliptic finite element problem. Math. Comp., 74:1-23, 2004.

[12] A. Ern and J. L. Guermond. Theory and Practice of Finite Elements. Sprigner-Verlag, New York, 2004.

[13] T. Ertekin, J. H. Abou-Kassem, and G. R. King. Basic Applied Reservoir Simulation. SPE textbook series, Vol. 7, Richardson, Texas, 2001.

[14] P. A. Forsyth. A control-volume, finite-element method for local mesh refinement in thermal reservoir simulation. SPE Reservoir Engineering, 5:561-566 (Paper SPE 18415), 1990.

[15] S. Günter and K. Lackner. A mixed implicit-explicit finite difference scheme for heat transport in magnetised plasmas. J. Comput. Phys., 228:282-293, 2009.

[16] S. Günter, K. Lackner, and C. Tichmann. Finite element and higher order difference formulations for modelling heat transport in magnetised plasmas. J. Comput. Phys., 226:2306-2316, 2007.

[17] S. Günter, Q. Yu, J. Kruger, and K. Lackner. Modelling of heat transport in magnetised plasmas using non-aligned coordinates. J. Comput. Phys., 209:354-370, 2005.

[18] J. Karátson and S. Korotov. Discrete maximum principles for finite element solutions of nonlinear elliptic problems with mixed boundary conditions. Numer. Math., 99:669-698, 2005.

[19] J. Karátson and S. Korotov. Discrete maximum principles for finite element solutions of some mixed nonlinear elliptic problems using quadratures. J. Comput. Appl. Math., 192:75-88, 2006.

[20] J. Karátson, S. Korotov, and M. Křížek. On discrete maximum principles for nonlinear elliptic problems. Math. Comput. Sim., 76:99-108, 2007.

[21] D. A. Karras and G. B. Mertzios. New PDE-based methods for image enhancement using SOM and Bayesian inference in various discretization schemes. Meas. Sci. Technol., 20:104012, 2009.

[22] D. Kuzmin, M. J. Shashkov, and D. Svyatskiy. A constrained finite element method satisfying the discrete maximum principle for anisotropic diffusion problems. J. Comput. Phys., 228:34483463, 2009.

[23] M. Křřžek and Q. Lin. On diagonal dominance of stiffness matrices in 3D. East-West J. Numer. Math., 3:59-69, 1995.

[24] C. Le Potier. Schéma volumes finis monotone pour des opérateurs de diffusion fortement anisotropes sur des maillages de triangles non structurés. C. R. Math. Acad. Sci. Paris, 341:787-792, 2005.

[25] C. Le Potier. A nonlinear finite volume scheme satisfying maximum and minimum principles for diffusion operators. Int. J. Finite Vol., 6:20, 2009. 
[26] C. Le Potier. Un schéma linéaire vérifiant le principe du maximum pour des opérateurs de diffusion très anisotropes sur des maillages déformés. C. R. Math. Acad. Sci. Paris, 347:105$110,2009$.

[27] F. W. Letniowski. Three-dimensional delaunay triangulations for finite element approximations to a second-order diffusion operator. SIAM J. Sci. Stat. Comput., 13:765-770, 1992.

[28] X. P. Li and W. Huang. An anisotropic mesh adaptation method for the finite element solution of heterogeneous anisotropic diffusion problems. J. Comput. Phys., (to appear):(arXiv:1003.4530v1).

[29] X. P. Li, D. Svyatskiy, and M. Shashkov. Mesh adaptation and discrete maximum principle for 2D anisotropic diffusion problems. Technical Report LA-UR 10-01227, Los Alamos National Laboratory, Los Alamos, NM, 2007.

[30] K. Lipnikov, M. Shashkov, D. Svyatskiy, and Yu. Vassilevski. Monotone finite volume schemes for diffusion equations on unstructured triangular and shape-regular polygonal meshes. $J$. Comput. Phys., 227:492-512, 2007.

[31] R. Liska and M. Shashkov. Enforcing the discrete maximum principle for linear finite element solutions of second-order elliptic problems. Comm. Comput. Phys., 3:852-877, 2008.

[32] M. J. Mlacnik and L. J. Durlofsky. Unstructured grid optimization for improved monotonicity of discrete solutions of elliptic equations with highly anisotropic coefficients. J. Comput. Phys., 216:337-361, 2006.

[33] D. Mumford and J. Shah. Optimal approximations by piecewise smooth functions and associated variational problems. Commun. Pure Appl. Math, 42:577-685, 1989.

[34] K. Nishikawa and M. Wakatani. Plasma Physics. Springer-Verlag Berlin Heidelberg, New York, 2000.

[35] P. Perona and J. Malik. Scale-space and edge detection using anisotropic diffusion. IEEE Trans. Pattern Anal. Mach. Intel., 12:629-639, 1990.

[36] P. Sharma and G.W. Hammett. Preserving monotonicity in anisotropic diffusion. J. Comput. Phys., 227:123-142, 2007.

[37] D. M. Y. Sommerville. An Introduction to the Geometry of $n$ Dimensions. Methuen \& Co. LTD., London, 1929.

[38] T.H. Stix. Waves in Plasmas. Amer. Inst. Phys., New York, 1992.

[39] G. Stoyan. On a maximum principle for matrices, and on conservation of monotonicity. With applications to discretization methods. Z. Angew. Math. Mech., 62:375-381, 1982.

[40] G. Stoyan. On maximum principles for monotone matrices. Lin. Alg. Appl., 78:147-161, 1986.

[41] G. Strang and G. J. Fix. An Analysis of the Finite Element Method. Prentice Hall, Englewood Cliffs, NJ, 1973. 
[42] R. S. Varga. On a discrete maximum principle. SIAM J. Numer. Anal., 3:355-359, 1966.

[43] J. Weickert. Anisotropic Diffusion in Image Processing. Teubner-Verlag, Stuttgart, Germany, 1998.

[44] J. Xu and L. Zikatanov. A monotone finite element scheme for convection-diffusion equations. Math. Comput., 69:1429-1446, 1999. 